This item was submitted to Loughborough's Research Repository by the author.

Items in Figshare are protected by copyright, with all rights reserved, unless otherwise indicated.

\title{
Fully automatic lesion boundary detection in ultrasound breast images
}

PLEASE CITE THE PUBLISHED VERSION

PUBLISHER

(C) 2007 SPIE

VERSION

VoR (Version of Record)

LICENCE

CC BY-NC-ND 4.0

REPOSITORY RECORD

Yap, Moi Hoon, Eran A. Edirisinghe, and Helmut E. Bez. 2019. "Fully Automatic Lesion Boundary Detection in Ultrasound Breast Images”. figshare. https://hdl.handle.net/2134/6499. 
This item was submitted to Loughborough's Institutional Repository (https://dspace.lboro.ac.uk/) by the author and is made available under the following Creative Commons Licence conditions.

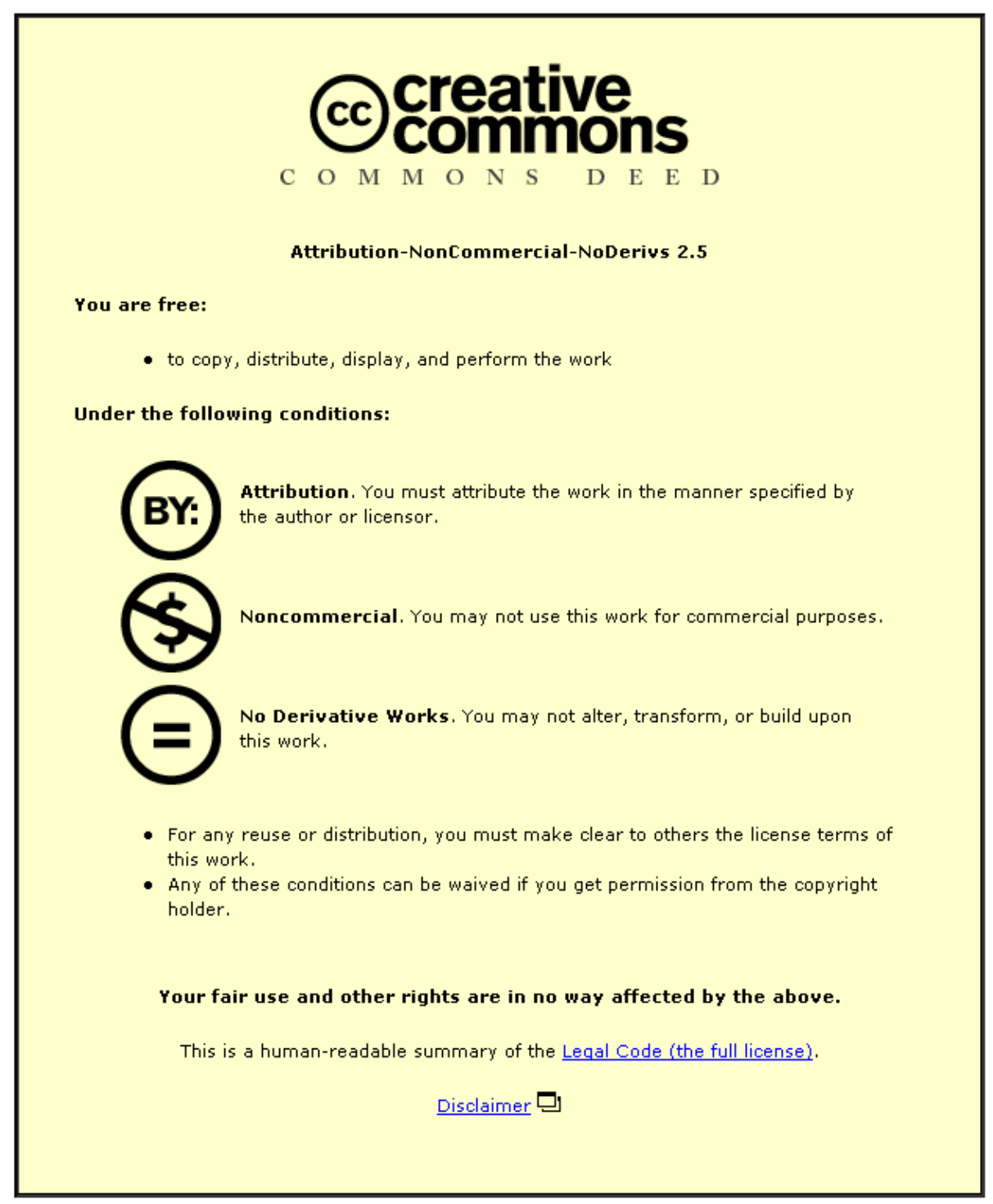

For the full text of this licence, please go to: http://creativecommons.org/licenses/by-nc-nd/2.5/ 


\title{
Fully Automatic Lesion Boundary Detection in Ultrasound Breast Images
}

\author{
M.H.Yap, E.A.Edirisinghe, H.E.Bez \\ Department of Computer Science, Loughborough University, UK.
}

\begin{abstract}
We propose a novel approach to fully automatic lesion boundary detection in ultrasound breast images. The novelty of the proposed work lies in the complete automation of the manual process of initial Region-of-Interest (ROI) labeling and in the procedure adopted for the subsequent lesion boundary detection. Histogram equalization is initially used to preprocess the images followed by hybrid filtering and multifractal analysis stages. Subsequently, a single valued thresholding segmentation stage and a rule-based approach is used for the identification of the lesion ROI and the point of interest that is used as the seed-point. Next, starting from this point an Isotropic Gaussian function is applied on the inverted, original ultrasound image. The lesion area is then separated from the background by a thresholding segmentation stage and the initial boundary is detected via edge detection. Finally to further improve and refine the initial boundary, we make use of a state-of-the-art active contour method (i.e. gradient vector flow (GVF) snake model). We provide results that include judgments from expert radiologists on 360 ultrasound images proving that the final boundary detected by the proposed method is highly accurate. We compare the proposed method with two existing stateof-the-art methods, namely the radial gradient index filtering (RGI) technique of Drukker et. al. and the local mean technique proposed by Yap et. al., in proving the proposed method's robustness and accuracy.
\end{abstract}

Keywords: Segmentation, Region-of-interest, multifractal, isotropic Gaussian, boundary detection, ultrasound imaging

\section{INTRODUCTION}

Breast cancer is one of the leading causes of death in females. According to the statistics reported in BBC news [1], one in every nine women in the UK will develop breast cancer in their life. Besides, in the USA, breast cancer affects one in every eight women. Further breast cancer is the leading cause of death of women in developed countries [2]. Fortunately, timely detection of breast cancer allows its effective treatment that could prevent mortality. As a result a number of computer-based breast cancer detection modalities have been developed in the past. It is widely accepted that the golden standard for breast screening and diagnostic is mammography, a special type of x-ray imaging used to create detailed images of the breasts [3]. Mammography has proven success in the early detection of cancer, considerably reducing cases of mastectomy and mortality. However, mammography creates a significant proportion of false positives and fails to detect in the subtle tissues and dense breasts.

Over the last two decades, a number of Computer Aided Diagnostic (CAD) tools have been developed to aid radiologists in detecting likely cases of breast cancer. CAD uses computer software to assist the film reader in identifying abnormalities on a mammogram; this is done by placing prompts over areas of concern. CAD techniques in medical imaging are developed for the automated differentiation between benign and malignant lesions. In some tools the technology provides functionality beyond straightforward computer-aided detection by providing cancer likelihood for a detected lesion, given image and/or patient characteristics [4].

Breast Screening in the UK is to be fully digitized within the next decade. Hence, development of complete CAD systems is crucial. At present ultrasound CAD systems require the identification of ROI and the initial marking of the centre of interest by an expert radiologist, before being able to automatically produce lesion boundaries. In this paper we propose an effective, fully automatic method for the point of interest selection of lesion ROI and for the further enhancement of lesion boundary detection.

For clarity of presentation this paper is divided into five sections. In section 2, we detail the motivation and background behind the proposed research, followed by the methodology in section 3. The experimental results are presented and discussed in section 4. Section 5 concludes with an insight to future directions of research.

Medical Imaging 2007: Image Processing, edited by Josien P. W. Pluim, Joseph M. Reinhardt, Proc. of SPIE Vol. 6512, 65123I, (2007) - 1605-7422/07/\$18 - doi: 10.1117/12.708625 


\section{BACKGROUND AND MOTIVATION}

A significant proportion of on-going research projects are focused towards creating Ultrasound Computer Aided Diagnosis (US CAD) tools with high sensitivity, high specificity, and high consistency, to name a few, Gurney 1994, Boone 1993, and Chen et al 2003 [5]. Unfortunately these systems are based on the assumption that the ROI will be preselected by the radiologist. For example, Chen et al managed to develop an accurate CAD system [5] in which the manual intervention by the physician to locate the ROI before the computer analysis, was required. Similarly the method proposed in [6] requires the lesion boundaries to be delineated manually.

Only two attempts have been proposed that have provided means for fully automatic lesion boundary detection that allows the tool to commence automatic processing from the original ultrasound image, rather than a human identified ROI. Drukker et al (2002) [7] was the first to propose the use of full (i.e. original) ultrasound images to automatically obtain the labeling of suspected lesions. To this effect Drukker et. al. investigated the use of the radial gradient index (RGI) filtering technique. It is common that in ultrasound images, lesions are almost invariably darker than the background. Thus in Drukker et al's work the gray-scale of the original ultrasound images are initially inverted. Subsequently images are pre-processed by a median filter to remove speckle noise and the resulting image is fed to a RGI filter. In RGI-filtering, the images are sub-sampled by a factor of 4 . The threshold for the RGI-filtered image is varied iteratively from 0.74 to 0.62 , until either at least one lesion of interest is detected. The detected areas with size smaller than $5 \mathrm{~mm}^{2}$ are discarded. Lesion candidates are segmented from the background by maximizing an average radial gradient (ARD) index for regions grown from the detected points. According to Drukker et al, maximizing the ARD index is more accurate than maximizing the RGI index [7]. At an overlap level of 0.4 with lesions outlined by a radiologist, $75 \%$ accuracy of lesion detection was reported.

In [8], Yap et. al. analysed the use of statistical methods (e.g., local mean) and values of fractal dimensions in initial lesion detection. Histogram equalization was used to pre-process the images followed by hybrid filtering and markercontrolled watershed segmentation. The minimum local mean and the minimum of the fractal dimensions of the identified segments were then used to determine the initial lesion. Subsequently neighbourhood segments were identified, which were finally combined to form the ROI. The accuracy of the automated ROI labeling is measured by an overlap of 0.4 with the lesion outline compared to the lesions labeled by the radiologist. It was reported that the accuracy of ROI detection when using local mean was $69.21 \%$, while fractal dimension was $54.21 \%$.

\section{METHODOLOGY}

Figure 1 illustrates the block diagram of the proposed approach. Stage 1 is to obtain the position of the region of interest. In stage 2, we obtain the initial contour of the tumor by using a Isotropic Gaussian function, and enhance the initial contour by using a special type of snake, namely, Gradient Vector Flow (GVF). The two main objectives of this paper are to investigate the effect of Isotropic Gaussian function in obtaining a more accurate initial boundary, and the use of GVF in boundary detection and segmentation. The details operation of the proposed algorithm will be presented in this section.

\subsection{Ultrasound Images}

Diagnostic Ultrasound (US) lies in the range of approximately 1 to 10 megahertz (MHz) [9]. The basic idea of diagnostic US is to transmit waves through the body and to be partially reflected at the interfaces between tissues [10]. The reflections form the US image, which are captured and recorded. US or Sonography is an important modality in the evaluation and treatment of breast masses. It's use is often tailored to the patient in answering a specific question/doubt that may have been raised in a pre examination involving mammography or physical means [11].

Imaging speckle is a phenomenon that occurs when a coherent source and a non-coherent detector are used to interrogate a medium, which is rough on the scale of the wavelength [12]. In another words, it is caused by the constructive and destructive interference of back-scattered signals due to unresolved tissue inhomogeneity. Due to this, speckle noise is a major limitation of ultrasound images, which affects human interpretation of the images as well as the accuracy of the Computer-assisted diagnostic techniques [13].

The test images used in our work are obtained from a professionally compiled Breast Ultrasound CD [14], which consists of an explanation and verifications from several qualified Radiologists. A total of 360 images from the Breast Ultrasound CD are selected for our experiments. 
Stage 1

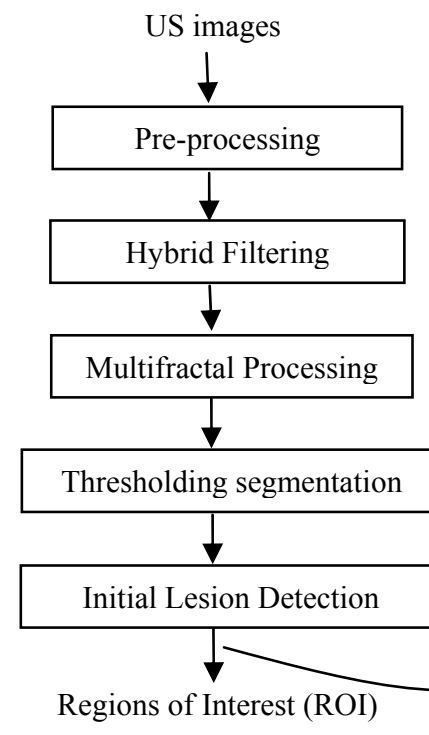

Stage 2

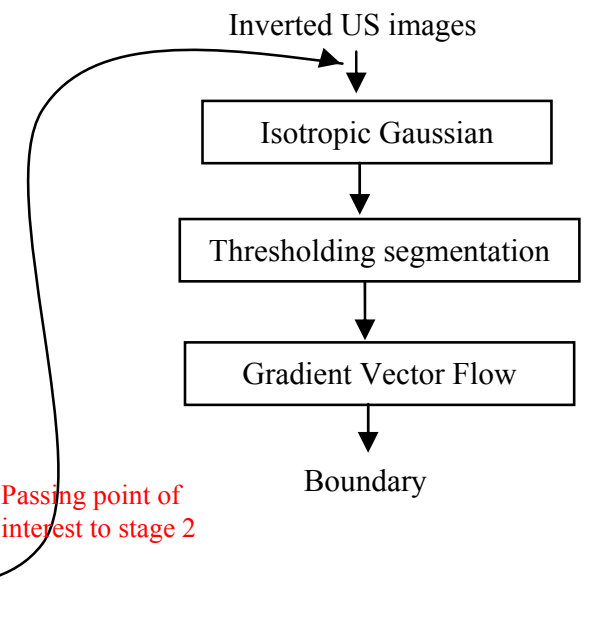

Figure 1. Overview of methodology

\subsection{Initial Lesion Detection}

One of the major problems in Ultrasound images is consistency. The quality of the breast ultrasound images is highly dependent on the scanner and the experience of the examiner. Hence, to maintain the consistency, a pre-processing step is crucial. To deal with the issue of guaranteeing the homogeneity of the original ultrasound images, we use histogram equalisation to achieve the above [15].

Subsequently a filtering stage is used to remove noise, which is a major obstacle for accurate segmentation of US images. In Yap et al's work of [15], a concept of Hybrid Filtering with multifractal enhancement was introduced. Hybrid filtering combines the strength of Nonlinear Diffusion filtering with the strength of linear filtering (Gaussian blur). It was shown that hybrid filtering produced better results than other filters used in the previous research. Further to enhance the results of hybrid filtering, multifractals with order -1 , was successfully used in the above work. A clear comparison between the use of different filters discussed above, has been provided in [15]. Within our present research context we use the work of [15] for the filtering stage.

We propose the use of a single fixed threshold to segment the image output from the preceding stage into ROI and nonROI regions. The result of the thresholding segmentation is analyzed by using a rule-based approach to perform the ROI selection. The first criterion used for the identification of lesions is the size of the segments. The suspected lesion, are identified as the larger segments out of the likely multiple segments that results from applying the single threshold segmentation. In addition to this, based on the additional guidance provided in the Ultrasound CD used to obtain our test data [14], 95\% of the tumours are located at the upper regions of the images. Hence, a reference point at

$x=\frac{\text { imageheight }}{3}$ and $y=\frac{\text { image width }}{2}$ is chosen. Figure 2 is the graphical illustration of stage 1 .

Based on our database, we managed to achieve an accuracy of $86 \%$ in initial lesion detection. This result is not ideal but it considerably good as compare to the two benchmark algorithms. 


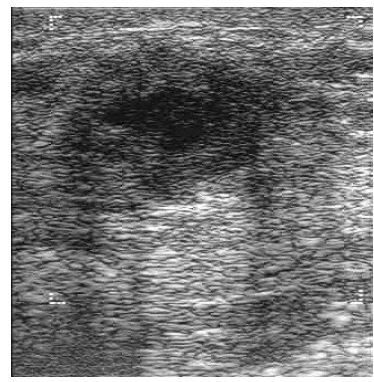

(a)

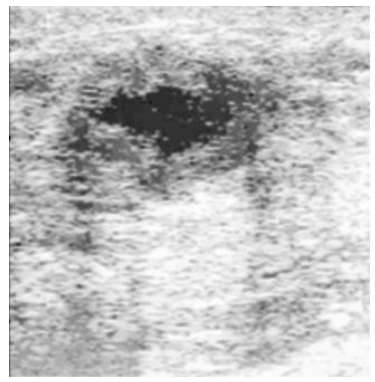

(b)

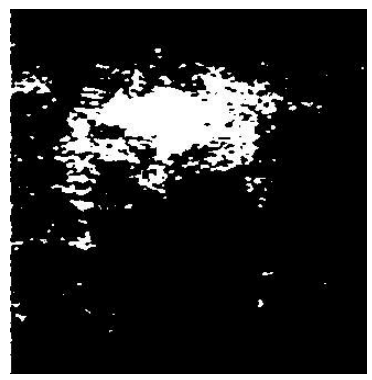

(c)

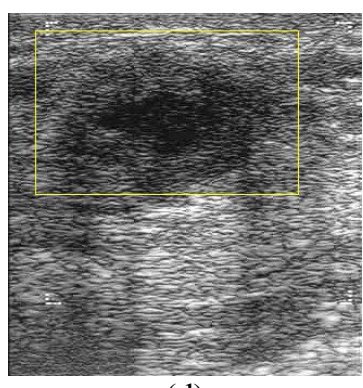

(d)

Figure 2. Output at stage 1: (a) Original image (b) After histogram equalization, hybrid filtering and multifractal enhancement (c) After thresholding segmentation (d) Labeling of region of interest (ROI)

\subsection{Initial Contour Detection}

In general, segmentation is a process used to divide an image into its constituent parts. It is credibly accepted that there are no standard segmentation methods that perform equally well in all images. In medical imaging, more specifically, breast ultrasound images, segmentation is an important intermediate stage of an ultrasound imaging system as the nature of the boundary of a lesion play an important role in differentiating between malignant and benign tumors [16]. In many CAD systems, a lesion segmentation step is initially used to obtain an accurate representation of the boundary of a tumor whose features are subsequently determined and used in lesion type classification [17]. According to Joo et al [17], the reason behind the inaccuracy of many existing ultrasound CAD systems is not the classification stage, but the segmentation stage. Even if corrective adjustments of the labeling were performed by the radiologists, obtaining the perfect boundary of the tumor lesions remains a challenge.

In order to incorporate the lesion shape into the creation of the partition, the inverted image $f(x, y)$ is multiplied by an isotropic Gaussian function centered at the seed point location $\left(\mu_{x}, \mu_{y}\right)$ from stage 1, with a fixed variance $\sigma_{c}^{2}$ as the constraint function [16]. The resulting image, $h(x, y)$ is given by:

$$
h(x, y)=f(x, y) N\left(x, y ; \mu_{x}, \mu_{y}, \sigma_{c}^{2}\right)
$$

The isotropic Gaussian images are segmented by thresholding segmentation with a fixed value of threshold. The initial boundary is obtained as illustrated in Figure 3.

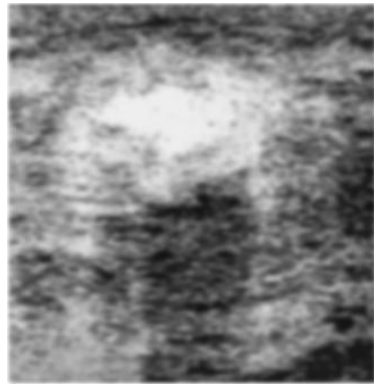

(a)

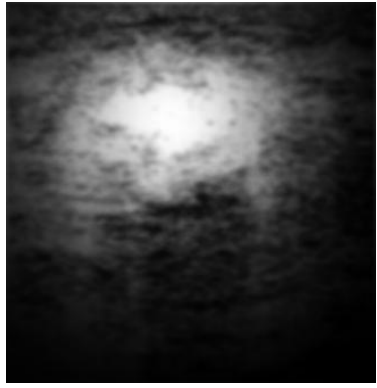

(b)

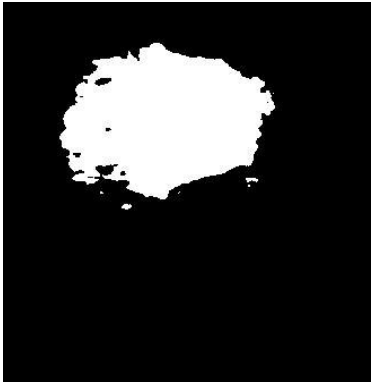

(c)

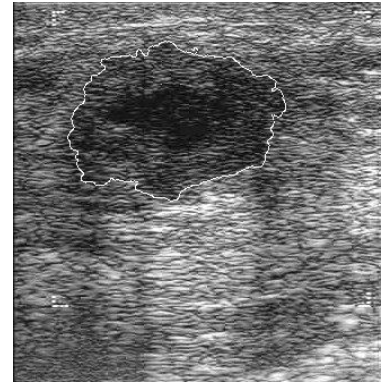

(d)

Figure 3. Output at stage 2: (a) Inverted image (b) Isotropic Gaussian on inverted US images (c) Thresholding segmentation (d) Initial boundary.

\subsection{Active Contour Models}

Deformable models, snakes, or Active contour models are curves defined within an image domain that can move under the influence of internal forces within the curve and external forces computed from the image data [18]. Yezzi et al. [19] applied the deformable model to segment cysts in ultrasound breast images. In Chen et. al.'s [20] research, the initial contour was detected, followed by the implementation of an active contour model to improve the segmentation results.

The traditional active contour model consists of two key difficulties. The first problem is the initial contour must be close to the exact boundary, otherwise it may converge to the wrong result. The second problem is that active contours have difficulties processing into boundary concavities [21]. Xu and Prince [22] introduced the gradient vector flow 
(GVF) which they claimed can resolve the above problem. From their definition of GVF in [22], the gradient vector flow field to be the vector field $\mathbf{v}(x, y)=[u(x, y), v(x, y)]$ that minimizes the energy functional

$$
\varepsilon=\iint \mu\left(\mu_{x}^{2}+\mu_{y}^{2}+v_{x}^{2}+v_{y}^{2}\right)+|\nabla f|^{2}|v-\nabla f|^{2} d x d y
$$

Using the calculus of variations, it has been shown that the GVF field can be found by solving the Euler Equations [22]:

$$
\begin{aligned}
& \mu \nabla^{2} u-\left(u-f_{x}\right)\left(f_{x}^{2}+f_{y}^{2}\right)=0 \\
& \mu \nabla^{2} v-\left(v-f_{y}\right)\left(f_{x}^{2}+f_{y}^{2}\right)=0
\end{aligned}
$$

where $\nabla^{2}$ is the Laplacian operator. The details of the GVF concept and its implementation are well-explained in [21, 22].

The gradient vector flow (GVF) algorithm is a free source available in Internet provided by Xu and Prince [21]. We performed our experiments on the initial boundary using the above implementation. The enhancement of the boundary can be clearly illustrated as in figure 4 .

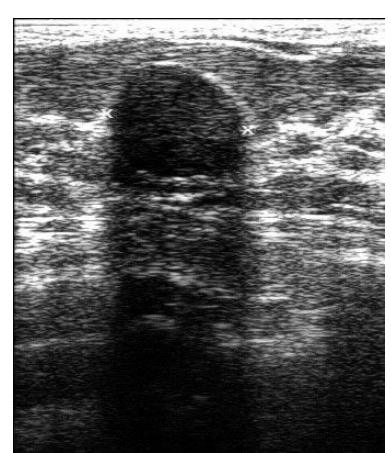

(a)

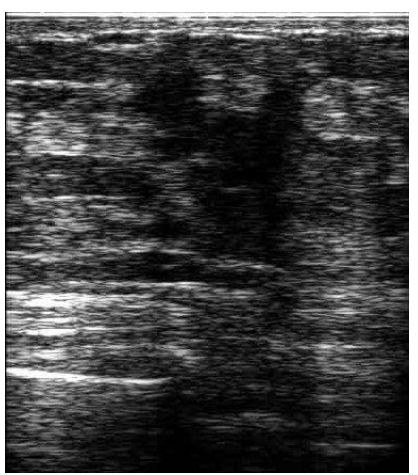

(d)

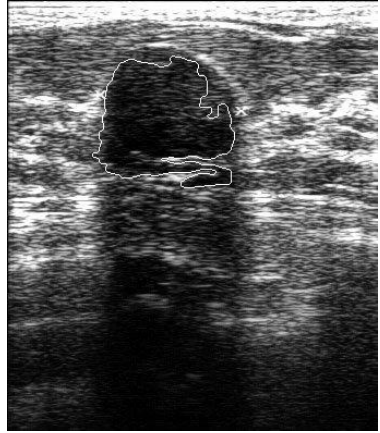

(b)

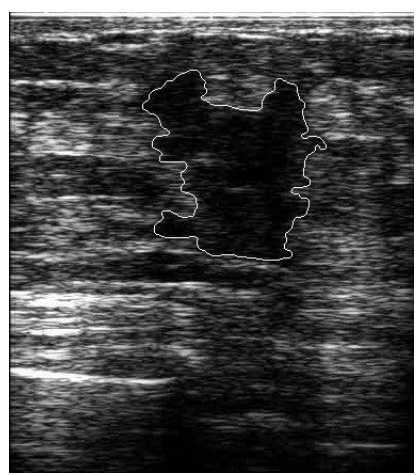

(e)

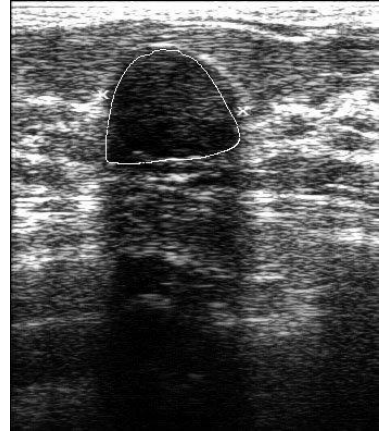

(c)

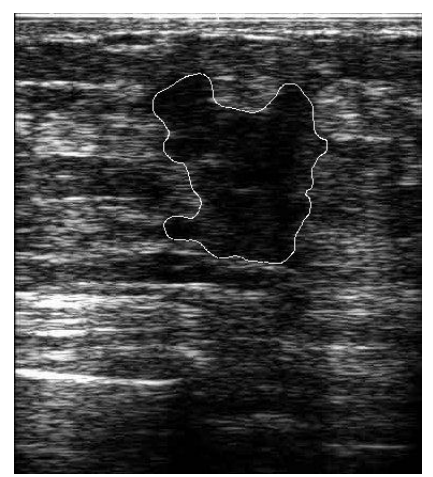

(f)

Figure 4. (a),(d) Original image (b),(e) Initial boundary (c),(f) Final boundary by gradient vector flow.

\section{RESULTS}

In figure 5 we illustrate the results obtained when the proposed system is used. In figure 6 we compare the results of the proposed algorithm with that of Drukker et al's and Yap et al's algorithms. A visual comparison of the results illustrated in figure 6 (given the diagnostic results in the Breast Ultrasound CD [14]) demonstrates that the proposed method performs better as compared to the benchmark algorithms.

Table 1 summarises the novelty of the work presented in this paper. It is noted however that fully automated functionality will not completely replace the role of a radiologist in detecting tumours. Nevertheless it provides an alternative way to save the radiologist's time in picking up the suspected regions manually. Thought it will improve the functionality of the CAD system, the medical expert/radiologist will take the final decision as to whether or not a tumour 
has been detected. Another contribution of the work is that it reserves the local and global details. The local details are referred to as the density of the tumor, boundary, width, height, and size of the tumors, while the global details are referred to as the shadowing effects, hyper-echoic, hypo-echoic, and the speckles noise on the full images. The local details are important in the classification of tumours. However with experience some radiologists may learn to identify tumours by only using the global details. Hence, it is important to reserve the global details.

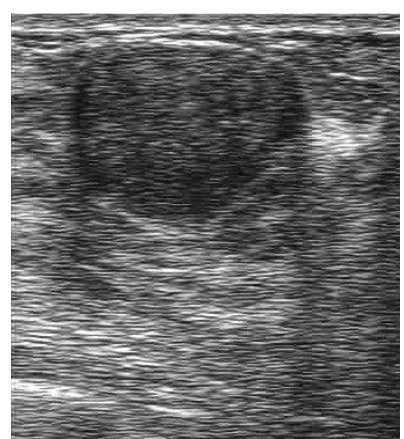

(a)

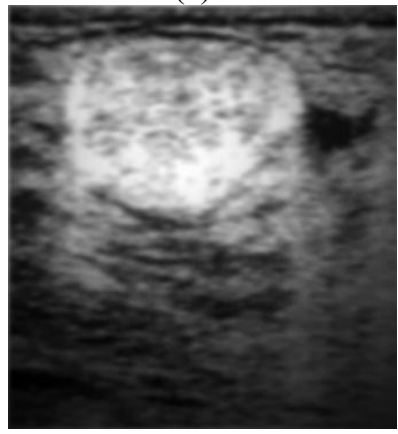

(e)

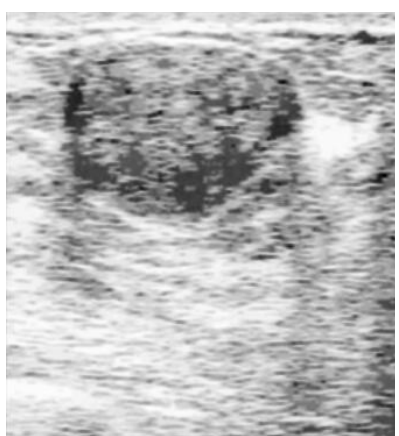

(b)

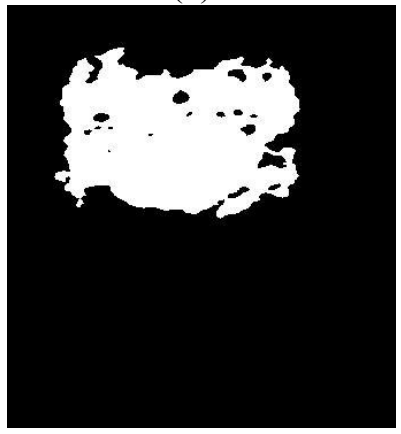

(f)

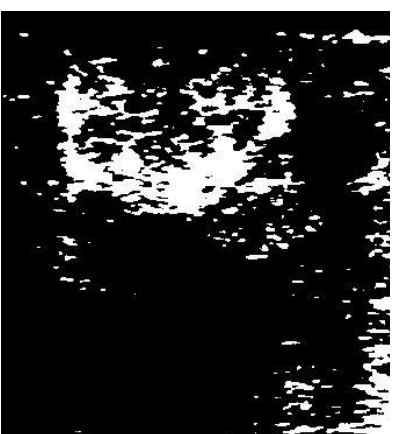

(c)

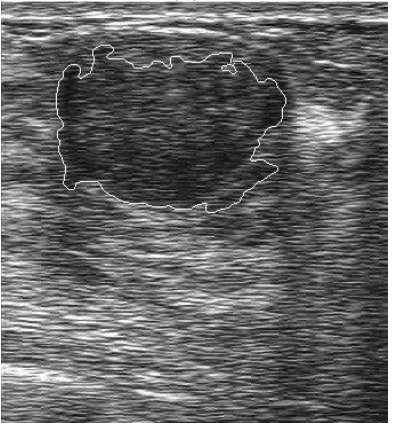

(g)

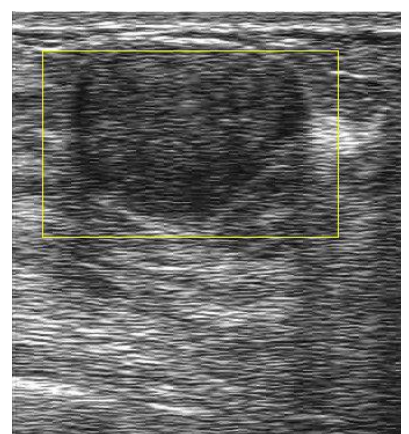

(d)

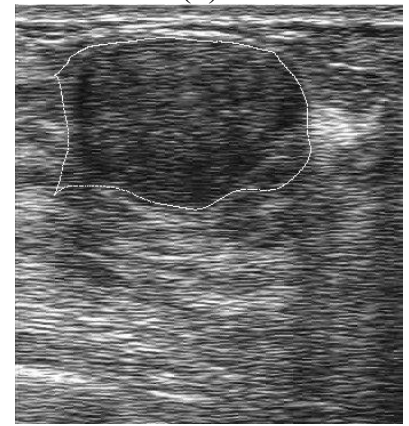

(h)

Figure 5. Output at various stages (fig.1): (a) Original image (b) After histogram equalization, hybrid filtering and multifractal enhancement (c) After thresholding segmentation (d) Labeling of region of interest (ROI) (e) Isotropic Gaussian on inverted US images (f) Thresholding segmentation (g) Initial boundary (h) Final boundary.

Table 1. Novelty of the proposed work

\begin{tabular}{|l|l|l|}
\hline Operation & Current CAD methods & Proposed method \\
\hline ROI Detection & Manually selected by radiologist & Fully automated selection \\
\hline $\begin{array}{l}\text { Segmentation/ } \\
\text { Boundary }\end{array}$ & $\begin{array}{l}\text { The boundary is shown on the } \\
\text { cropped ROI. Therefore decisions } \\
\text { that would need the consideration } \\
\text { of global image details cannot be } \\
\text { made. The global dimension of the } \\
\text { image (context) will also be lost. }\end{array}$ & $\begin{array}{l}\text { The boundary is detected from the full ultrasound } \\
\text { images, which maintain the local details as well as } \\
\text { global details. }\end{array}$ \\
& &
\end{tabular}




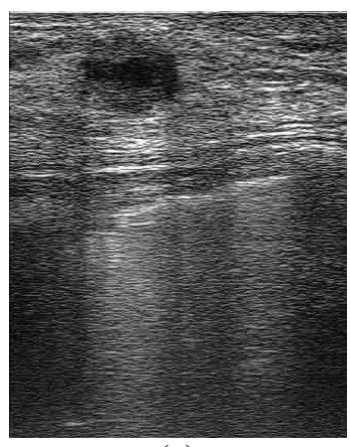

(a)

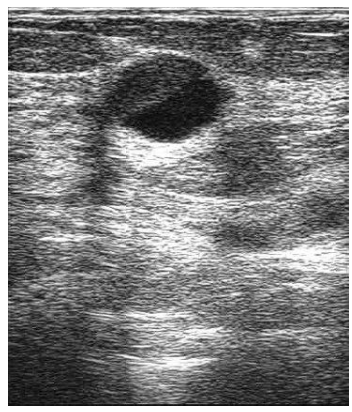

(e)

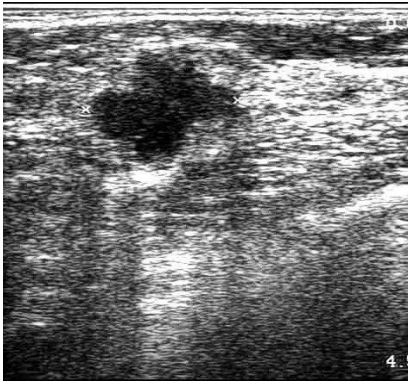

(i)

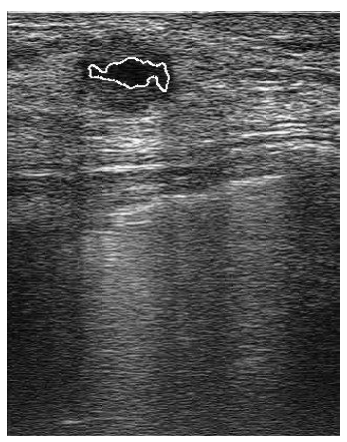

(b)

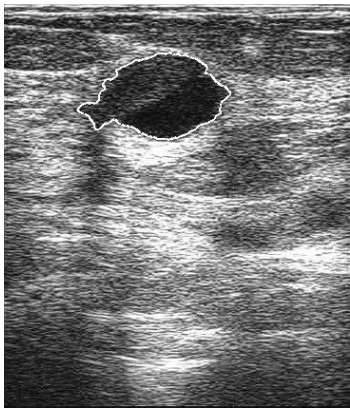

(f)

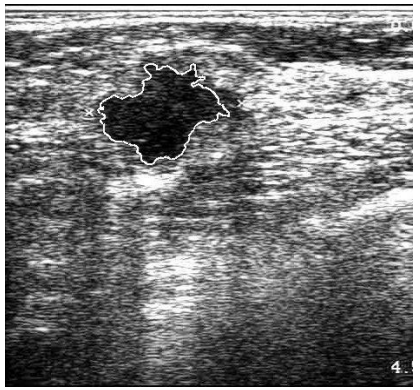

(j)

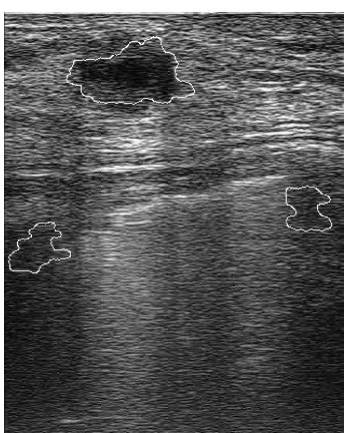

(c)

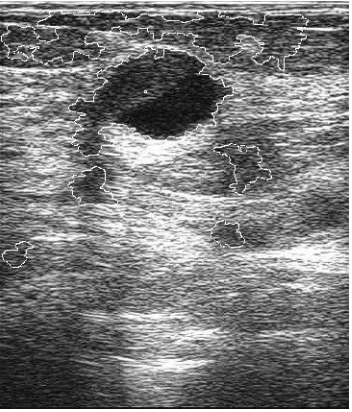

(g)

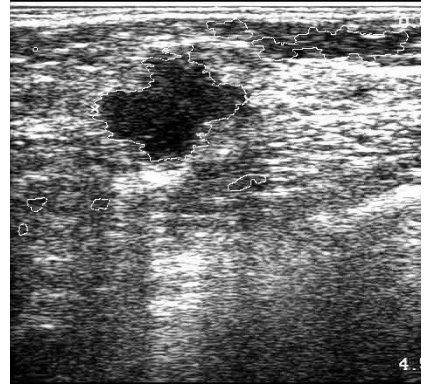

(k)

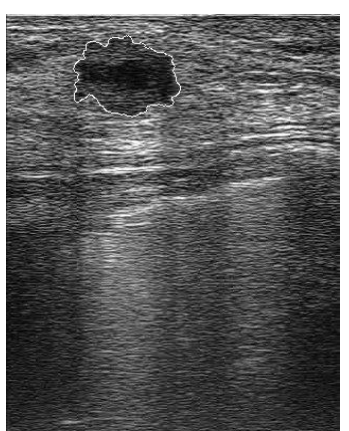

(d)

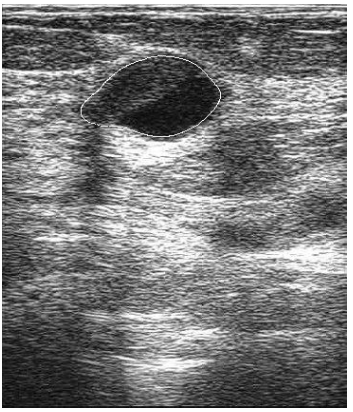

(h)

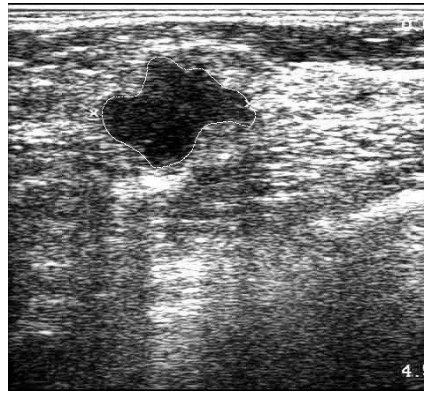

(1)

Figure 6. Visual performance comparison with benchmark algorithms. (a),(e),(i) The original images. (b),(f),(j) The result of Drukker et al's algorithm. (c),(g),(k) The result of Yap et al's algorithm. (d),(h),(l) The result of proposed method.

\section{CONCLUSION}

We have proposed a fully automatic procedure for lesion ROI detection, centre-of-interest capture and lesion boundary detection. We have compared it with current state-of-the-art techniques and have proved the approach's effectiveness in fully automatic lesion boundary detection on un-cropped ultrasound images.

In future, we will improve the proposed algorithm into a more adaptive algorithm, for instance, the system will be able to automatically select the optimum parameter set for the snake model. Further, we propose to investigate the application of implicit active contour models, or level sets, in ultrasound images. We are currently working on proposing effective classification methods for separating benign and malignant tumors, with the hope of delivering a complete, fully automated Ultrasound Computer-Aided Diagnosis system (US CAD) for breast cancer screening and detection. 


\section{REFERENCES}

1. Story from BBC News, "Breast Cancer," vol. 2006, pp. 1, Friday, 30 January 2004, 11:04 GMT. 2004.

2. D.R. Chen, R.F. Chang, and Y.L. Huang, "Breast Cancer Diagnosis using self-organizing map for sonography," Ultrasound in Med. \& Biol., Vol. 26, No. 3, pp. 405-411, 2000.

3. "Imaginis - General information on Mammography". 2007(01/03).

4. M. Kallergi, "Computer-aided Diagnosis of mammographic microcalcification clusters," Med. Phys, vol. 31, pp. 314-326, 2004.

5. D. R. Chen, R. F. Chang, W. M. Chen and W. K. Moon, "Computer-aided diagnosis for 3-dimensional breast ultrasonography," Arch. Surg., vol. 138, pp. 296-302, Mar. 2003.

6. C. M. Chen, Y. H. Chou, K. C. Han, G. S. Hung, C. M. Tiu, H. J. Chiou and S. Y. Chiou, "Breast lesions on sonograms: computer-aided diagnosis with nearly setting-independent features and artificial neural networks," Radiology, vol. 226, pp. 504-514, Feb. 2003.

7. K. Drukker, M. L. Giger, K. Horsh, M. A. Kupinski and C. J. Vyborny. Computerized lesion detection on breast ultrasound. Med. Physics 29(7), pp. 1438-1446.

8. M.H.Yap, H.T.Ewe. "Region of Interest (ROI) detection in Ultrasound Breast Images". Proc. MMU International Symposium on Information and Communication Technologies (M2USIC), 2005.

9. S. C. Bushong, Diagnostic Ultrasound. United States: McGraw-Hill, 1999, pp. 152.

10. P. Suetens, Fundamental of Medical Imaging. Cambridge University Press, 2002, pp. 294.

11. L. A. Venta, C. M. Dudiak, C. G. Salomon and M. E. Flisak, "Sonographic Evaluation of the Breast," RadioGraphics, vol. 14, pp. 29-50, 1994.

12. A. Achim, A. Bezerianos and P. Tsakalides, "Novel Bayesian Multiscale Method for Speckle Removal in Medical Ultrasound Images," IEEE Transactions on Medical Imaging, vol. 20, pp. 772-783, 2001.

13. K. Z. Abd-Elmoniem, A. M. Youssef and Y. M. Kadah. (2002, Real-time speckle reduction and coherence enhancement in ultrasound imaging via nonlinear anisotropic diffusion. IEEE Transactions on Biomedical Engineering 49(9), pp. 997-1014.

14. S.T. Prapavesis, B.D. Fornage, C.F. Weismann, A. Palko, P. Zoumpoulis, et al. Breast Ultrasound and US-Guided Interventional Techniques. A multimedia Teaching File, $1^{\text {st }}$ edition. Thessaloniki Greece, (2001).

15. M.H. Yap, E.A. Edirisinghe, H.E. Bez. "Object Boundary Detection in Ultrasound Breast Images", Third Canadian Robotics and Machine Vision Conference (CRV 2006).

16. M. Kupinski, M.L. Giger, P. Lu, and Z. Huo, "Computerized detection of mammographic lesions: Performance of artificial neural network with enhanced feature extraction," in SPIE, 2434:598-605, 1995.

17. S. Joo, Y.S.Yang, W.K. Moon, and H.C.Kim, "Computer-Aided Diagnosis of Solid Breast Nodules: Use of an Artificial Neural Network Based on Multiple Sonographic Features," IEEE Trans. On Med. Imaging, 23(10), Oct. 2004.

18. C. Xu and J. L. Prince, "Snakes, Shapes, and Gradient Vecto Flow," IEEE Transactions on Image Processing, vol. 7, pp. 359-369, 1998.

19. A. Yezzi, S. Kichenassamy, A. Kumar, P. Olver and A. Tannenbaum, "A geometric snake model for segmentation of medical imagery," IEEE Trans on Med Imaging, vol. 16, pp. 199-209, 1997.

20. Y.L. Huang, and D.R. Chen, "Support Vector Machines in sonography application to decision making in the diagnosis of breast cancer," Journal of Clinical Imaging 29(2005): 179-184.

21. C. Xu and J.L. Prince, "Gradient vector flow: A new external force for snakes," IEEE Proc. Conf. on Computer Vision and Pattern Recognition, 1997, pp. 66-71.

22. C. Xu and J.L. Prince, "Snakes, Shapes, and Gradient Vector Flow," IEEE Transactions on Image Processing, Vol. 7, No. 3, March 